\title{
Analysis and Experiments for High-Efficiency Class-F and Inverse Class-F Power Amplifiers
}

\author{
Young Yun Woo, Youngoo Yang, Member, IEEE, and Bumman Kim, Senior Member, IEEE
}

\begin{abstract}
This paper presents analytic and experimental comparisons for high-efficiency class-F and inverse class-F amplifiers. The analytic formula of the efficiencies, output powers, dc power dissipations, and fundamental load impedances of both amplifiers are derived from the ideal current and voltage waveforms. Based on the formula, the performances are compared with a reasonable condition: fundamental output power levels of class-F and inverse class-F amplifiers are conditioned to be identical. The results show that the inverse class-F amplifier has better efficiency than that of class-F amplifiers as the on-resistance of the transistor increases.

For experimental comparison, we have designed and implemented the class-F and inverse class-F amplifiers at 1-GHz band using a GaAs MESFET and analyzed the measured performances. Experimental results shows $10 \%$ higher power-added efficiency of the inverse class-F amplifier than that of the class-F amplifier, which verifies the waveform analysis.
\end{abstract}

Index Terms-Class-F amplifier, harmonics control circuit, highefficiency amplifier, inverse class-F amplifier, power amplifier.

\section{INTRODUCTION}

A GROWING popularity of the wireless communication systems makes the high-efficiency RF power amplifiers very important RF components. The class-F amplifier, which has short load termination at even-order harmonics (current peaking) and open load termination at odd-order harmonics (voltage peaking), has become a representative of the high-efficiency amplifier [1]-[3]. Very recently, the inverse class-F amplifier has started to draw attention due to its superior performance. It is commonly known that the inverse class-F amplifiers, which have open load at even-order harmonics (voltage peaking) and short load at odd-order harmonics (current peaking), can deliver higher efficiency than class-F operation. Some papers with partial analyses, simulations, or experiments, which demonstrated advantages of the inverse class-F amplifier, have been reported [4]-[6]. However, there have been no reports treating fully analytic and experimental comparisons for the clear explanation of the better efficiency of the inverse class-F amplifier.

Manuscript received November 24, 2005; revised February 1, 2006. This work was supported in part by the Korean Ministry of Education under the BK21 Project and by the Center for Broadband OFDM Mobile Access (BrOMA) at the Pohang University of Science and Technology (POSTECH) under the ITRC Program of the Korean MIC, supervised by IITA (IITA-2005-C1090-0502-0008).

Y. Y. Woo and B. Kim are with the Department of Electrical Engineering, Pohang University of Science and Technology, Pohang 790-784, Korea (e-mail: w0yun@ postech.ac.kr).

Y. Yang is with the School of Information and Communication, Sungkyunkwan University, Suwon 440-746, Korea (e-mail: yang09@skku. edu).

Digital Object Identifier 10.1109/TMTT.2006.872805

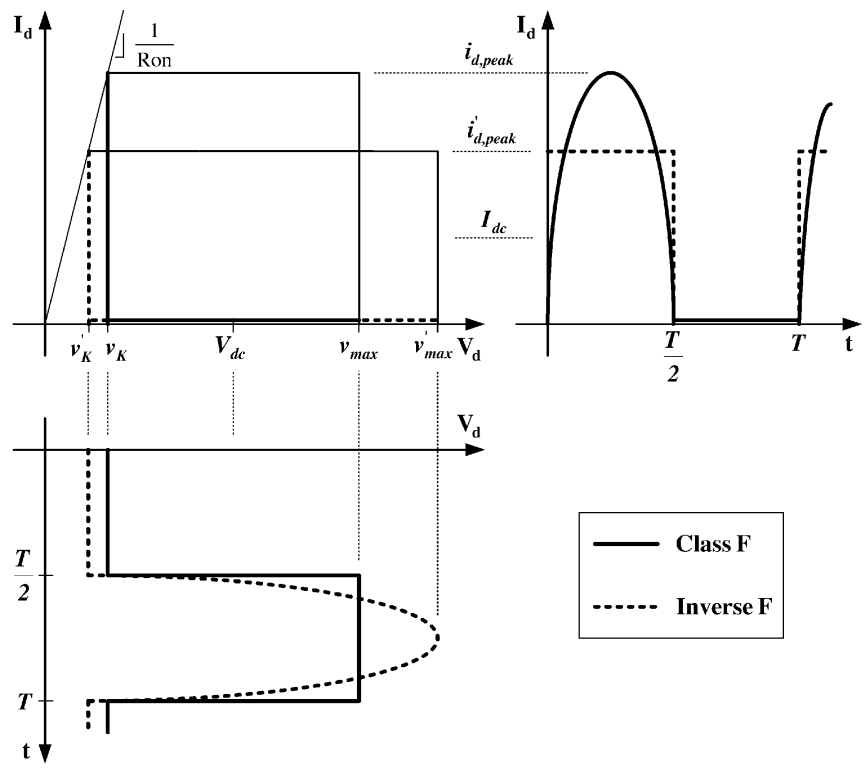

Fig. 1. Ideal current and voltage waveforms according to the load line on transistor's $I-V$ plane. Class-F (solid line). Inverse class-F (dotted line).

The purpose of this paper is to provide the quantitative and clear explanation of better efficiency, and the design guide for optimizing the output power or efficiency of the inverse class-F amplifiers in comparison to the class-F amplifiers. For that purpose, the efficiency equations of the inverse class-F and conventional class-F amplifiers are derived using ideal time-domain waveforms. The analytic comparisons are then carried out using the equations under the condition that the fundamental output power of both amplifiers are identical around where they have maximum PAE.

For an experimental comparison, the class- $\mathrm{F}$ and inverse class-F amplifiers at $1-\mathrm{GHz}$ band are designed and implemented. The analysis and experimental results clearly show why the inverse class-F amplifier has higher efficiency than the class-F amplifier.

\section{ANALYTIC APPROACH}

\section{A. Representation of Parameters}

Fig. 1 shows the ideal time-domain current and voltage waveforms of the class-F and inverse class-F amplifiers, when they have the same fundamental output power under the same drain biases. The class-F amplifiers have half-sinusoidal current and square-wave voltage signals. On the contrary, the inverse class-F amplifiers have square-wave current and half-sinusoidal voltage signals. These ideal waveforms of the class-F and inverse class-F amplifiers can be analyzed using Fourier series 
expansion, which gives expressions for the various parameters, such as a de power dissipation, a fundamental RF output power, and a required fundamental load impedance to obtain a proper RF output power. The calculation is summarized in the Appendix. For the class-F amplifiers, they are presented using the variables shown in Fig. 1 as follows:

$$
\begin{aligned}
P_{\mathrm{dc}} & =I_{\mathrm{dc}} \cdot V_{\mathrm{dc}}=\frac{i_{d, \text { peak }}}{\pi} V_{\mathrm{dc}} \\
P_{\text {fund }} & =\frac{i_{d, \text { peak }}}{\pi}\left(V_{\mathrm{dc}}-R_{\mathrm{on}} \cdot i_{d, \text { peak }}\right) \\
R_{L, \text { fund }} & =-\frac{V_{\text {fund }}}{I_{\text {fund }}}=\frac{8 \cdot\left(V_{\mathrm{dc}}-R_{\mathrm{on}} \cdot i_{d, \text { peak }}\right)}{\pi \cdot i_{d, \text { peak }}} .
\end{aligned}
$$

In the same way, parameters of the inverse class-F amplifiers can be calculated as in the following equations using $V_{\mathrm{dc}}$, $i_{d, \text { peak }}^{\prime}$, and $R_{\text {on }}$ :

$$
\begin{aligned}
P_{\mathrm{dc}}^{\prime} & =I_{\mathrm{dc}}^{\prime} \cdot V_{\mathrm{dc}}=\frac{i_{d, \text { peak }}^{\prime}}{2} V_{\mathrm{dc}} \\
P_{\text {fund }}^{\prime} & =\frac{i_{d, \text { peak }}^{\prime}}{2}\left(V_{\mathrm{dc}}-R_{\mathrm{on}} \cdot i_{d, \text { peak }}^{\prime}\right) \\
R_{L, \text { fund }}^{\prime} & =-\frac{V_{\text {fund }}^{\prime}}{I_{\text {fund }}^{\prime}}=\frac{\pi^{2} \cdot\left(V_{\mathrm{dc}}-R_{\mathrm{on}} \cdot i_{d, \text { peak }}^{\prime}\right)}{4 \cdot i_{d, \text { peak }}^{\prime}}
\end{aligned}
$$

where $R_{\text {on }}=v_{K}^{\prime} / i_{d, \text { peak }}^{\prime}$.

Using (1), (2), (4), and (5), the efficiencies of the class-F and inverse class-F amplifiers can be easily calculated as follows:

$$
\begin{gathered}
\eta=100 \frac{\left(V_{\mathrm{dc}}-R_{\mathrm{on}} \cdot i_{d, \text { peak }}\right)}{V_{\mathrm{dc}}}(\%) \\
\eta^{\prime}=100 \frac{\left(V_{\mathrm{dc}}-R_{\mathrm{on}} \cdot i_{d, \text { peak }}^{\prime}\right)}{V_{\mathrm{dc}}}(\%)
\end{gathered}
$$

where $\eta$ and $\eta^{\prime}$ are the efficiencies of the class-F and inverse class-F amplifiers, respectively. From (7) and (8), if $R_{\text {on }}$ is zero, the efficiencies of the class-F and inverse class- $F$ amplifiers are $100 \%$. The efficiencies could be differentiated from each other due to the different knee voltages originated from the different peak current levels.

\section{B. Performance Comparison}

To compare the performances of the class- $\mathrm{F}$ and inverse class-F amplifiers, we take the condition of the same output power at the same drain bias voltage. Since the same transistors are used in the design, other design parameters may need to be adjusted in order to satisfy the above conditions for both amplifiers.

Using the condition of $P_{\text {fund }}=P_{\text {fund }}^{\prime}$ from (2) and (5), we can get a second-order equation of $i_{d, \text { peak }}^{\prime}$ for a fixed drain bias and $i_{d, \text { peak }}$ as follows:

$$
\begin{aligned}
R_{\mathrm{on}} \cdot i_{d, \text { peak }}^{\prime}- & V_{\mathrm{dc}} \cdot i_{d, \text { peak }}^{\prime}\left(V_{\mathrm{dc}}-R_{\mathrm{on}} \cdot i_{d, \text { peak }}\right) \\
\pi & +\frac{2 \cdot i_{d, \text { peak }}}{\pi}
\end{aligned}
$$

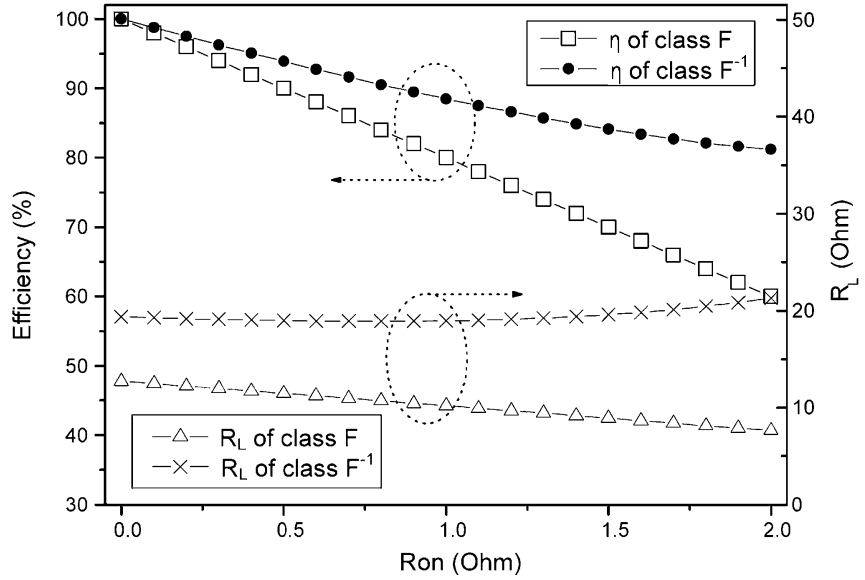

(a)

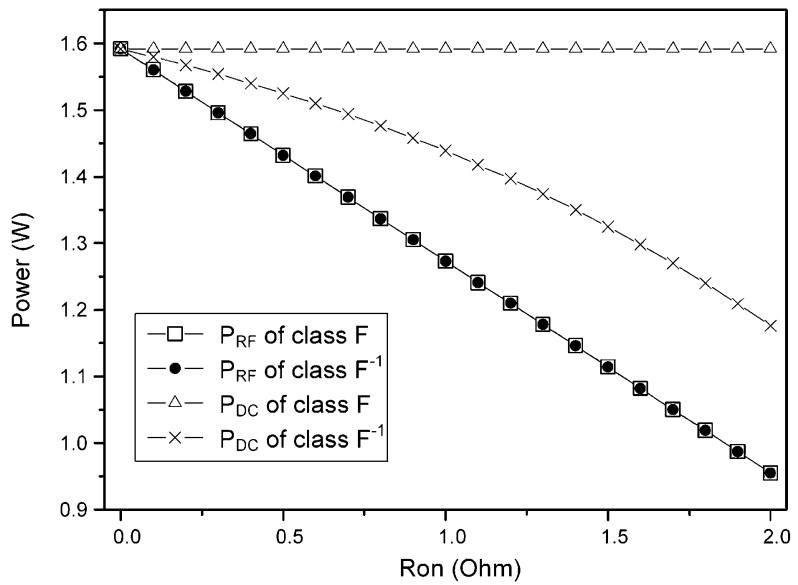

(b)

Fig. 2. Performances of the class-F and inverse class-F amplifiers for the identical fundamental RF output power condition with varying $R_{\text {on }}$ from 0 to $2 \Omega$. (a) Efficiency and fundamental load impedance. (b) DC power dissipation and fundamental RF output power.

The solution of (9) can be represented as

$$
=\frac{V_{\mathrm{dc}} \pm \sqrt{V_{\mathrm{dc}}^{2}-\frac{8 \cdot R_{\mathrm{on}} \cdot i_{d, \text { peak }}}{\pi}\left(V_{\mathrm{dc}}-R_{\mathrm{on}} \cdot i_{d, \text { peak }}\right)}}{2 \cdot R_{\mathrm{on}}} .
$$

Selecting a reasonable value of $i_{d, \text { peak }}^{\prime}$ from (10) and adding the $R_{\text {on }}=0$ case, (10) is rewritten as

$$
\begin{aligned}
& i_{d, \text { peak }}^{\prime} \\
& =\left\{\begin{array}{l}
\frac{2}{\pi} i_{d, \text { peak }}, \quad \text { for } R_{\mathrm{on}}=0 \\
\frac{V_{\mathrm{dc}}-\sqrt{V_{\mathrm{dc}}^{2}-\frac{8 \cdot R_{\mathrm{on}} \cdot i_{d, \text { peak }}}{\pi}\left(V_{\mathrm{dc}}-R_{\mathrm{on}} \cdot i_{d, \text { peak }}\right)}}{2 \cdot R_{\text {Fon }}}, \\
\text { for } R_{\mathrm{on}} \neq 0 .
\end{array}\right.
\end{aligned}
$$

If (11) is substituted to the inverse class-F formula of (4)-(6) and (8), we can easily obtain the analytic forms of dc power dissipation, RF output power, load impedance, and efficiency 


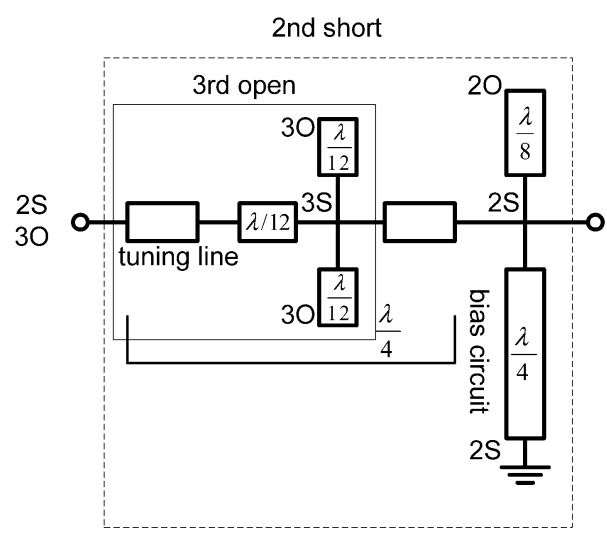

(a)

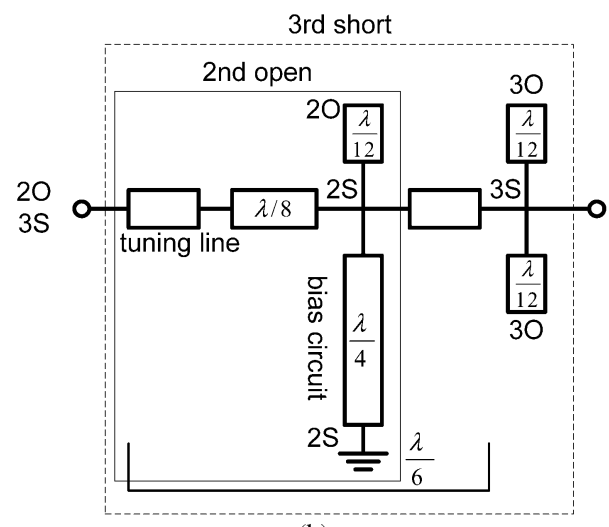

(b)

Fig. 3. Harmonic control circuits for: (a) class-F amplifier and (b) inverse class-F amplifier.

of the inverse class-F amplifier as functions of not $i_{d, \text { peak }}^{\prime}$, but $i_{d, \text { peak }}$, where the RF output power is identical with that of the class-F amplifier. The resulting equations are not presented in this paper because of complexity. The calculation is numerically performed using MATLAB.

The comparison results of performances between the class- $F$ and inverse class-F amplifiers are presented in Fig. 2. For the calculation, we assumed a $V_{\mathrm{dc}}$ supply of $5 \mathrm{~V}$ and $i_{d \text {,peak }}$ of $1 \mathrm{~A}$ with a uniform transconductance.

The efficiency of the inverse class- $\mathrm{F}$ amplifier is better than that of the class-F amplifier with increasing $R_{\text {on }}$ due to the higher $V_{\text {peak }}$ to $V_{K}$ ratio to maintain an identical $P_{\text {fund }}$, as shown in Fig. 2(a). Fig. 2(b) shows a significantly decreasing dc power consumption of the inverse class-F amplifier for the same RF output power, as $R_{\text {on }}$ increases. From the analysis results and Fig. 1, we can expect that the inverse class-F amplifier delivers superior efficiency when the amplifiers are not limited by the breakdown voltage, but by the bias voltage, which is the normal operation condition of handset power amplifiers.

\section{DESIGN AND EXPERIMENTS FOR THE VERIFICATION}

For experimental comparison of the class- $\mathrm{F}$ and inverse class-F amplifiers, we have designed 1-GHz-band class-F and inverse class-F amplifiers using OKI's 0.1-W MESFET KGF1284. The simulation of the two amplifiers is performed using Agilent's Advanced Design System (ADS) with an in-house MESFET large-signal model [7].
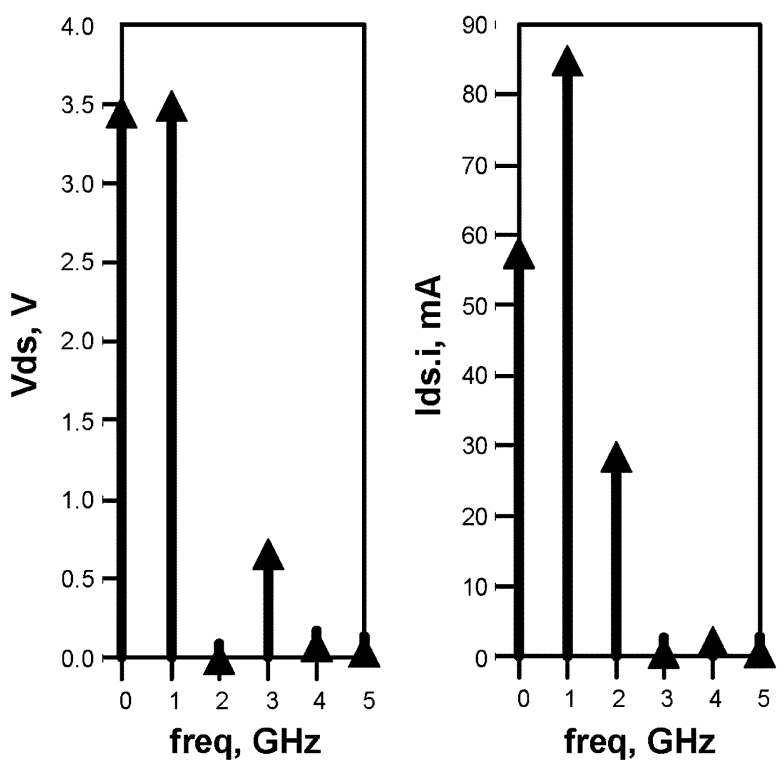

(a)
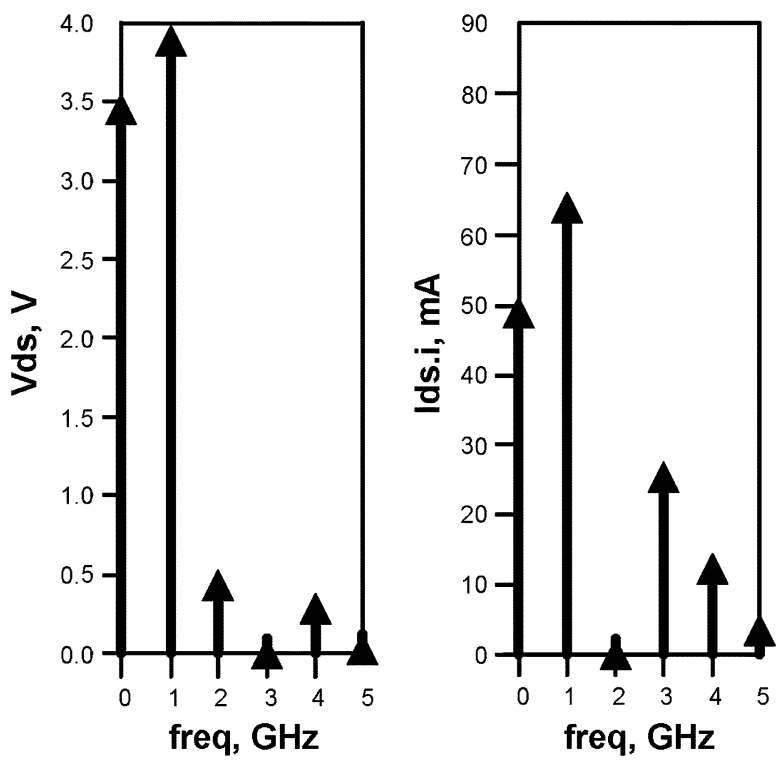

(b)

Fig. 4. Simulated root-mean-square values of drain voltage and current signals in frequency domain for: (a) class-F amplifier and (b) inverse class-F amplifier.

The designed class-F and inverse class-F amplifiers have the same input matching networks and the same dc bias (class-B bias point with $V g=-2.4 \mathrm{~V}$ and $V d=3.6 \mathrm{~V}$ ), except operation modes (class-F versus inverse class-F). The second and third harmonic components are terminated properly in the design for circuit simplicity.

In order to control the two harmonic components, we have constructed the output harmonic control networks, as illustrated in Fig. 3. Fig. 3(a) and (b) shows the harmonic control circuits for class-F and inverse class-F amplifiers, respectively. The control circuits include both arm shunt stubs for better harmonic trap and tuning lines (gray lines in Fig. 3) for compensating detuning effects of the device's parasitic passive components.

Output matching networks of the two amplifiers are designed for optimum performance with these harmonic control circuits. We have simulated the two designed amplifiers and probed 


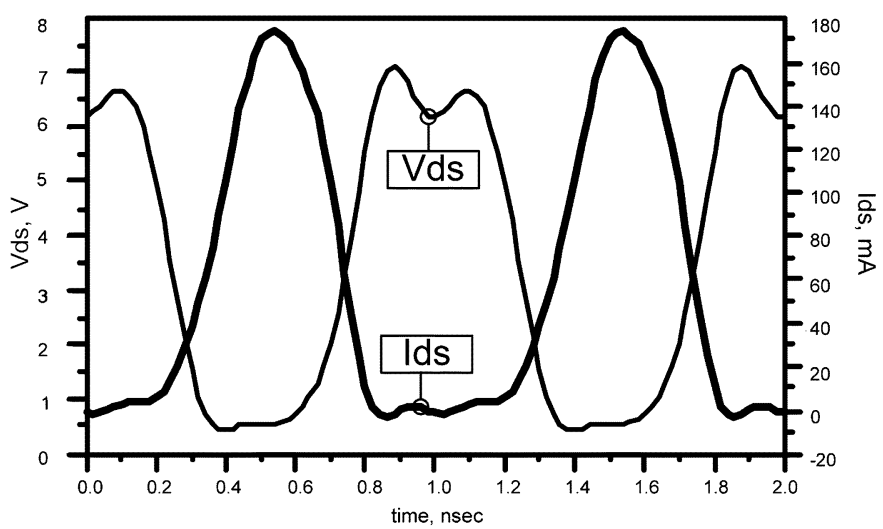

(a)

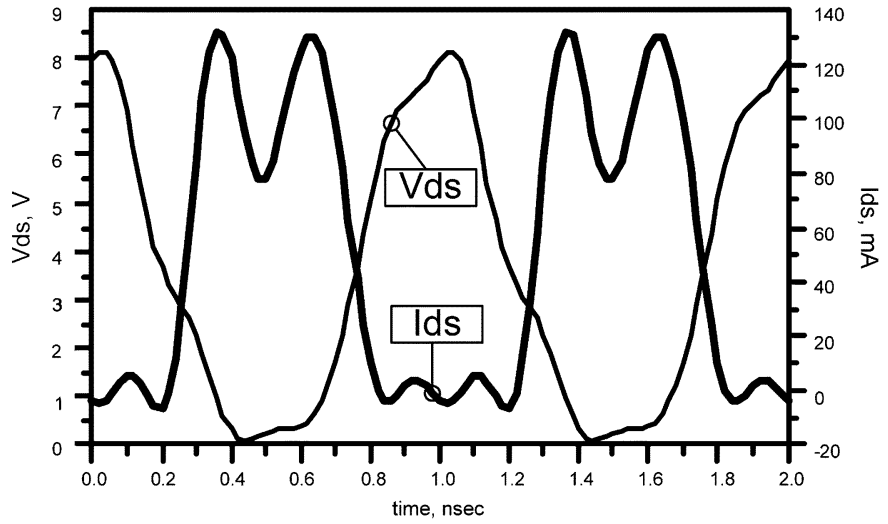

(b)

Fig. 5. Simulated time-domain voltage and current waveforms for: (a) class-F amplifier and (b) inverse class-F amplifier.

voltage and current signals. The results are shown in Figs. 4 and 5, which are the drain voltages and currents of the two amplifiers in the frequency and time domains, respectively.

As expected, the class-F amplifier has third-order voltage peaking with third-order load impedance of $252.26 \Omega$ and second-order current peaking with second-order load impedance of $1.05 \Omega$, while the inverse class-F amplifier has second-order voltage peaking with second-order load impedance of $419.5 \Omega$ and third-order current peaking with third-order load impedance of $3.00 \Omega$, as shown in Fig. 4. Time-domain signals also show that the class-F amplifier has a square-like voltage signal and a current signal close to half-sine wave, while the inverse class-F amplifier has the opposite (see Fig. 5).

The photographs of the implemented class-F and inverse class-F amplifiers are shown in Fig. 6. We have measured output powers and power-added efficiencies (PAEs) of the two amplifiers using a 1-GHz one-tone signal. Fig. 7 shows the simulated and measured power responses and PAEs of the class-F and inverse class-F amplifiers. As shown in Fig. 7(a), the inverse class-F amplifier has approximately 1-dB lower gain because the square-wave drain current needs more input drive than the half-sinusoidal drain current for the same output power level. The maximum PAE of the class-F amplifier is approximately $64 \%$ at an output power of $22.5 \mathrm{dBm}$. The maximum PAE of the inverse class-F amplifier is approximately $74 \%$ at the same output power level, which is $10 \%$ higher than that of the class-F amplifier. The superior PAE performance of the inverse class-F

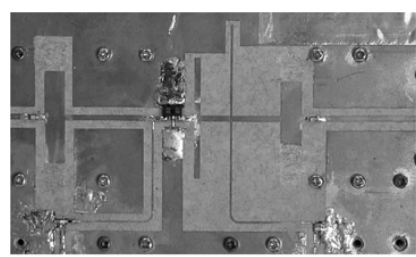

(a)

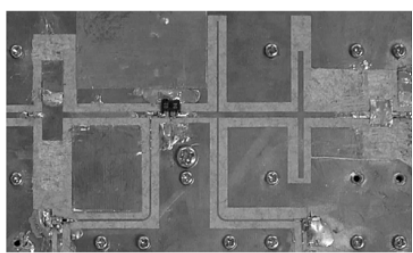

(b)
Fig. 6. Implemented: (a) class-F amplifier and (b) inverse class-F amplifier.

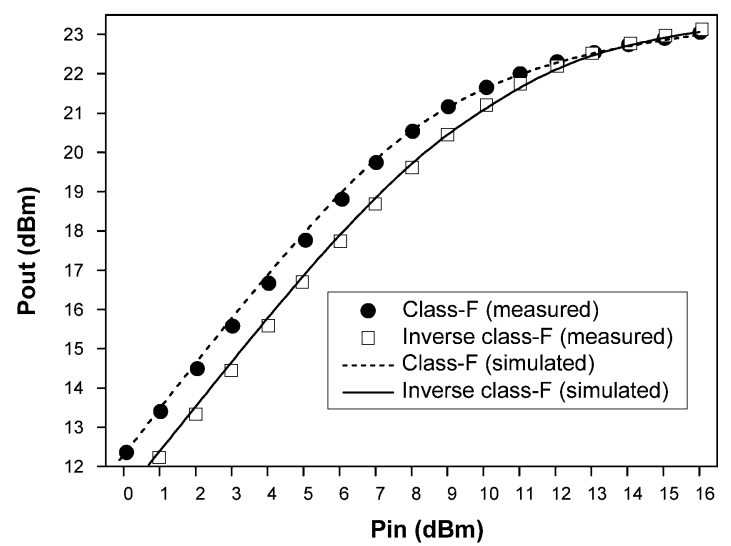

(a)

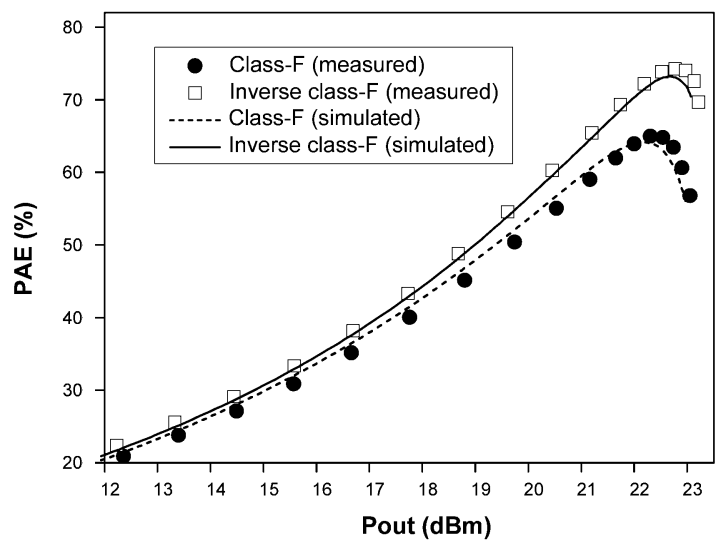

(b)

Fig. 7. Simulated and measured performances of the class-F and inverse class-F amplifiers. (a) Output power levels. (b) PAEs.

amplifier at the same output power level supports the analysis results well.

\section{CONCLUSION}

We have analyzed and compared the time-domain waveforms of the class-F and inverse class-F amplifiers. The analysis results show that, under the operation condition of the same drain bias, the inverse class-F amplifier has superior PAE to the class-F amplifier when on-resistance $\left(R_{\mathrm{on}}\right)$ of the transistor exists. As $R_{\text {on }}$ gets larger or the drain bias voltage gets lower, the performance difference also increases. For experimental comparison, we have designed and implemented $1-\mathrm{GHz}$ class-F and inverse class-F amplifiers. The implemented inverse class-F amplifier has a maximum PAE of approximately $74 \%$ at $22.7-\mathrm{dBm}$ output power, which is $10 \%$ higher than that of the class-F amplifier. This result clearly validates our analysis. 


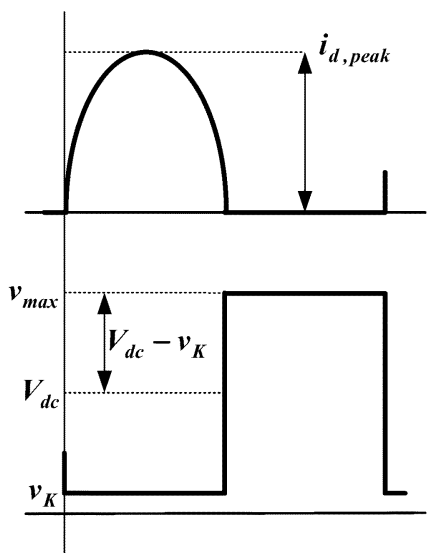

(a)

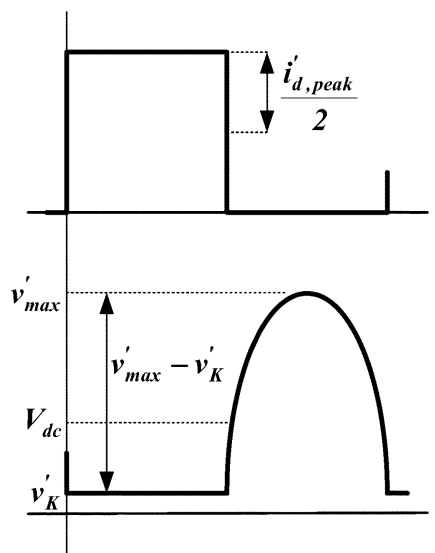

(b)
Fig. 8 Ideal current and voltage waveforms for: (a) class-F amplifier and (b) inverse class-F amplifier.

From the analysis and experiment for the comparison of the two amplifiers, the inverse class-F amplifier is expected to be useful to the power amplifiers for the base-stations or handsets requiring high efficiency. This study also provides a good design guide for the inverse class-F amplifier to extract higher efficiency.

\section{APPENDIX}

\section{A. Class-F}

Fig. 8 shows the ideal current and voltage waveforms of the conventional class-F amplifiers. To extract each frequency component, the current and voltage waveforms of Fig. 8 are expanded using a Fourier series as follows:

$i_{D}=i_{d, \text { peak }}\left(\frac{1}{\pi}+\frac{1}{2} \sin \omega_{0} t-\frac{2}{\pi} \sum_{n=2,4,6, \ldots} \frac{1}{n^{2}-1} \cos n \omega_{0} t\right)$

$v_{D}=V_{\mathrm{dc}}-\frac{4 \cdot\left(V_{\mathrm{dc}}-v_{k}\right)}{\pi} \sum_{1,3,5, \ldots} \frac{1}{n} \sin n \omega_{0} t$

where $i_{D}$ and $v_{D}$ are the time-domain current and voltage waveforms including $\mathrm{dc}$ and RF components, respectively. $R_{\text {on }} \cdot i_{d \text {,peak }}$ can be substituted for the knee voltage $v_{K}$ (see Fig. 1).

The current and voltage waveforms can then be rearranged with each harmonic's component. The dc and fundamental RF components of (A.1) and (A.2) are separated as the functions of peak current, dc voltage, and $R_{\mathrm{on}}$ as

$$
\begin{aligned}
I_{\mathrm{dc}} & =\frac{i_{d, \text { peak }}}{\pi} \\
I_{\text {fund }} & =\frac{i_{d, \text { peak }}}{2} \sin \omega_{0} t \\
V_{\text {fund }} & =-\frac{4 \cdot\left(V_{\mathrm{dc}}-R_{\text {on }} \cdot i_{d, \text { peak }}\right)}{\pi} \sin \omega_{0} t .
\end{aligned}
$$

\section{B. Inverse Class-F Amplifiers}

Fig. 8 shows the ideal current and voltage waveforms of the inverse class-F amplifiers. The current and voltage waveforms are expanded using the Fourier series as follows:

$$
\begin{aligned}
i_{D}^{\prime}= & \frac{i_{d, \text { peak }}^{\prime}}{2}\left(1+\frac{4}{\pi} \sum_{n=1,3,5, \ldots} \frac{1}{n} \sin n \omega_{0} t\right) \\
v_{D}^{\prime}= & V_{k}^{\prime}+\left(v_{\max }^{\prime}-v_{k}^{\prime}\right) \\
& \cdot\left(\frac{1}{\pi}-\frac{1}{2} \sin \omega_{0} t+\frac{2}{\pi} \sum_{n=2,4,6, \ldots} \frac{1}{n^{2}-1} \cos n \omega_{0} t\right) .
\end{aligned}
$$

$R_{\text {on }} \cdot i_{d, \text { peak }}^{\prime}$ can be substituted to the knee voltage $v_{K}^{\prime}$. From (A.7), $v_{\max }^{\prime}$ can be written using dc supply voltage as follows:

$$
v_{\max }^{\prime}=\pi \cdot V_{\mathrm{dc}}-(\pi-1) \cdot R_{\mathrm{on}} \cdot i_{d, \text { peak }}^{\prime} .
$$

Here, the current and voltage waveforms can be rearranged with each harmonic's component. The dc and fundamental RF components of (A.6) and (A.7) are separated as the functions of peak current, dc voltage, and $R_{\mathrm{on}}$ as

$$
\begin{aligned}
I_{\mathrm{dc}}^{\prime} & =\frac{i_{d, \text { peak }}^{\prime}}{2} \\
I_{\text {fund }}^{\prime} & =\frac{2 \cdot i_{d, \text { peak }}^{\prime}}{\pi} \sin \omega_{0} t \\
V_{\text {fund }}^{\prime} & =-\frac{\pi \cdot\left(V_{\mathrm{dc}}-R_{\mathrm{on}} \cdot i_{d, \text { peak }}^{\prime}\right)}{2} \sin \omega_{0} t .
\end{aligned}
$$

\section{REFERENCES}

[1] F. H. Raab, "Class-E, class-C, and class-F power amplifiers based upon a finite number of harmonics," IEEE Trans. Microw. Theory Tech., vol. 49, no. 8, pp. 1462-1468, Aug. 2001.

[2] A. N. Rudiakova and V. G. Krizhanovski, "Driving waveforms for class-F power amplifiers," in IEEE MTT-S Int. Microw. Symp. Dig., Jun. 2000, pp. 473-476.

[3] M. K. Kazimierczuk, "A new concept of class F tuned power amplifier," in Proc. 27th Midwest Circuits Syst. Symp., 1984, pp. 425-428.

[4] A. Inoue, A. Ohta, S. Goto, T. Ishikawa, and Y. Matsuda, "The efficiency of class-F and inverse class-F amplifiers," in IEEE MTT-S Int. Microw. Symp. Dig., Jun. 2004, pp. 1947-1950.

[5] C. J. Wei, P. DiCarlo, Y. A. Tkachenko, R. McMorrow, and D. Bartle, "Analysis and experimental waveform study on inverse class-F mode of microwave power FETs," in IEEE MTT-S Int. Microw. Symp. Dig., Jun. 2000, pp. 525-528.

[6] S. Goto, "Effect of bias condition and input harmonic termination on high efficiency inverse class-F amplifiers," in Proc. IEEE Eur. Microw. Conf., Sep. 2001, vol. 3, pp. 113-116.

[7] T. M. Roh, Y. Kim, Y. Suh, W. S. Park, and B. Kim, "A simple and accurate MESFET channel-current model including bias-dependent dispersion and thermal phenomena," IEEE Trans. Microw. Theory Tech. vol. 45, no. 8, pp. 1252-1255, Aug. 1997.

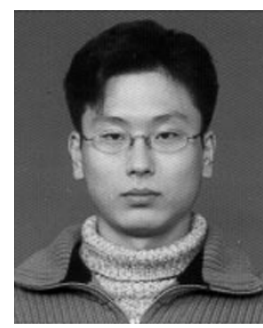

Young Yun Woo received the B.S. degree in electrical and computer engineering from Han-Yang University, Seoul, Korea, in 2000, and is currently working toward the Ph.D. degree in electronic and electrical engineering from the Pohang University of Science and Technology (POSTECH), Pohang, Korea.

His current research interests include RF power amplifier design, linear power amplifier (LPA) system design, and digital predistortion (DPD) techniques for linearizing high power amplifiers. 


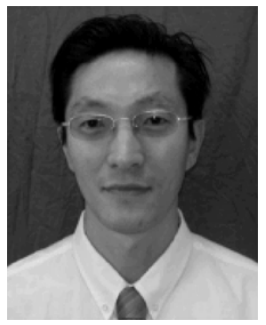

Youngoo Yang (S'99-M'02) was born in Hamyang, Korea, in 1969. He received the Ph.D. degree in electrical and electronic engineering from the Pohang University of Science and Technology (POSTECH), Pohang, Korea, in 2002.

From 2002 to 2005, he was with Skyworks Solutions Inc., Newbury Park, CA, where he designed power amplifiers for various cellular handsets. Since March 2005, he has been with the School of Information and Communication, Sungkyunkwan University, Suwon, Korea, where he is currently an Assistant Professor. His research interests include design of power amplifiers, RF integrated-circuit (RFIC) design, and modeling of high power amplifiers or devices.

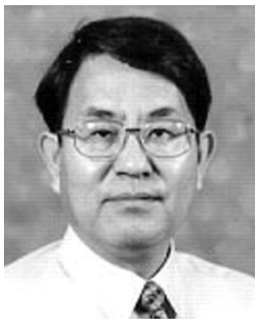

Bumman Kim (S'77-M'78-SM'97) received the $\mathrm{Ph} . \mathrm{D}$. degree in electrical engineering from Carnegie-Mellon University, Pittsburgh, PA, in 1979.

From 1978 to 1981 , he was engaged in fiber-optic network component research with GTE Laboratories Inc. In 1981, he joined the Central Research Laboratories, Texas Instruments Incorporated, where he was involved in development of GaAs power field-effect transistors (FETs) and monolithic microwave integrated circuits (MMICs). He has developed a largesignal model of a power FET, dual-gate FETs for gain control, high-power distributed amplifiers, and various millimeter-wave MMICs. In 1989, he joined the Pohang University of Science and Technology, Pohang, Korea, where he is a Professor with the Electronic and Electrical Engineering Department and Director of the Microwave Application Research Center, where he is involved in device and circuit technology for RFICs. In 2001, he was a Visiting Professor of electrical engineering with the California Institute of Technology, Pasadena. He has authored over 150 published technical papers.

Dr. Kim is a member of the Korean Academy of Science and technology and Academy of Engineering of Korea. He is an associate editor for the IEEE TRANSACTIONS ON MICROWAVE THEORY AND TECHNIQUES. 